\title{
COMPARACIÓN DE MÉTODOS DE DESCARGA PARA VERTIDOS DE SALMUERAS, PROVENIENTES DE UNA PLANTA DESALINIZADORA EN SONORA, MÉXICO
}

\author{
Germán Eduardo DÉVORA ISIORDIA*, Adriana ROBLES LIZÁRRAGA, \\ Gustavo Adolfo FIMBRES WEIHS y Jesús ÁLVAREZ SÁNCHEZ
}

Departamento de Ciencias del Agua y Medio Ambiente, Instituto Tecnológico de Sonora. Calle 5 de febrero 818 sur, Ciudad Obregón, Sonora, México, C. P. 85000

*Autor para correspondencia: german.devora@itson.edu.mx

(Recibido julio 2015; aceptado septiembre 2016)

Palabras clave: ósmosis inversa, agua de rechazo, impacto ambiental

\section{RESUMEN}

Una alternativa para hacer frente al desabasto de agua para consumo humano, industrial y agrícola, es la desalinización de aguas marinas y salobres. Este proceso consiste en la separación de sales del agua mediante diversas tecnologías. Sin embargo, a pesar de los beneficios que ofrecen, una desventaja importante del proceso es la cantidad de salmuera generada y su impacto ambiental como resultado de su alta salinidad. Bajo este contexto, el objetivo de este estudio es realizar una comparación de técnicas de descarga para vertidos de salmueras producidas por una planta desalinizadora. Se propuso llevar a cabo la investigación de métodos de disposición, para proponer la técnica más adecuada que disminuya el impacto ambiental producido en la zona de estudio. Para ello, se operó una planta desalinizadora de $100 \mathrm{~m}^{3} / \mathrm{d}$ con recuperación del $85 \%$ en agua producto y $15 \%$ en agua de rechazo. Se comparó la diferencia de concentraciones de salinidad entre agua alimentación y rechazo (salmuera). Los resultados indican que hubo un aumento en la salinidad del agua rechazo respecto a la de alimentación (de $2931 \mathrm{mg} / \mathrm{L}$ hasta $6756 \mathrm{mg} / \mathrm{L}$ de sólidos disueltos totales). Este aumento en la concentración muestra que es importante regular o disponer el vertido de salmueras para evitar impactos negativos al ecosistema receptor. Para este proyecto el método más adecuado, según la zona de estudio, es operar una granja acuícola para producción de camarón con alto valor comercial. Esta propuesta se identificó para el control y el uso de vertidos de salmuera en la zona del Valle del Yaqui, Sonora, México.

Key words: reverse osmosis, rejection water, environmental impact

\begin{abstract}
One of the alternatives for tackling water shortages for human, industrial and agricultural use is desalination of seawater and brackish water. Desalination involves the separation of dissolved salts from water, by means of several technologies. However, despite the benefits that the technologies offer, an important disadvantage of the process is the amount of brine generated and its environmental impact as a result of its high salinity. In this context, the aim of this study is to compare the techniques available to discharge brines from a desalination plant, through the investigation of adequate disposal methods, in order to propose the best technique, to decrease the environmental
\end{abstract}


impact caused in the study site. For this purpose, a $100 \mathrm{~m}^{3} / \mathrm{d}$ desalination plant was operated at $85 \%$ product recovery and $15 \%$ retentate. The salinity concentration difference between the feed and retentate (brine) was compared. The results indicate an increase in the retentive salinity with respect to the feed water, from $2931 \mathrm{mg} / \mathrm{L}$ to 6756 $\mathrm{mg} / \mathrm{L}$ of total dissolved solids. This concentration increase shows that it is important to regulate the disposal of brine in order to avoid negative impacts on the receiving ecosystem. For this project, the most adequate method according to the study site was to operate an aquaculture farm for the production of high commercial value shrimp. Hence, this was the proposed integral brine discharge management in the zone of the Yaqui Valley in Sonora, Mexico.

\section{INTRODUCCIÓN}

La producción de agua potable se ha convertido en una preocupación a nivel mundial. Más de mil millones de personas no tienen acceso a agua potable y aproximadamente 2300 millones viven en regiones con escasez de agua (Morillo et al. 2014).

En México también se enfrentan severos problemas de abasto de agua ante la creciente demanda de la población por contar con el vital líquido. Dos terceras partes del país son zonas áridas o semiáridas, en ellas se concentra $77 \%$ de la población, se genera $84 \%$ de la actividad económica y se registra únicamente $28 \%$ de escurrimiento del agua (Dévora et al. 2012).

En cuanto al agua subterránea, la sobreexplotación ha generado intrusión salina y a nivel nacional ésta se presenta en 17 acuíferos costeros en los estados de Baja California Sur, Baja California, Sonora, Veracruz y Colima. En el Estado de Sonora, principalmente en la región suroeste, 450 pozos agrícolas han sido abandonados debido su alta salinidad, causada por intrusión salina dada su cercanía a la costa. Esto ha obligado a buscar otras fuentes de abastecimiento de agua dulce, entre ellas la desalinización de agua por ósmosis inversa (Dévora et al. 2012).

La desalinización se define como un proceso de separación de sales disueltas de aguas salobres o de mar para convertirlas en aguas adecuadas para consumo humano, uso industrial o agrícola (Medina 2000). Destacan los sistemas térmicos: destilación múltiple efecto (MED, por sus siglas en inglés), destilación por destello múltiple etapa (MSF, por sus siglas en inglés) y destilación solar (DS). En cuanto a los sistemas por membrana están: ósmosis inversa $(\mathrm{OI})$, electrodiálisis (ED) y nano filtración (NF) (Dévora et al. 2013).

Los problemas de disponibilidad de los recursos hídricos abandonados en el Valle del Yaqui, Sonora, se deben principalmente a la alta concentración de sales en los pozos (Dévora et al. 2013). Es por esto que la desalinización de aguas salobres para su aplicación en agricultura ha creado grandes expectativas como forma alternativa para conseguir recursos hídricos de calidad en las zonas más castigadas por la escasez de agua.

Aunque el uso de tecnologías de desalinización de agua de mar a nivel mundial ha aumentado significativamente en las últimas décadas y ha mostrado muchos beneficios en diferentes actividades humanas, se ha prestado poca atención al impacto del subproducto principal (agua de rechazo o salmuera) en los ecosistemas y especies (Gacía y Ballesteros 2001, Lattemann y Höpner 2007, Morillo et al. 2014). Este subproducto contiene principalmente los componentes naturales del agua de entrada, en forma concentrada. Además, la salmuera, en concentraciones por encima de $35000 \mathrm{mg} / \mathrm{L}$ de sólidos disueltos totales (SDT), contiene aditivos y productos de corrosión. Los aditivos son productos químicos utilizados para el control de la contaminación biológica (por ejemplo cloro), control escala de incrustaciones (anti-incrustantes), la reducción de la espuma y la inhibición de la corrosión. Dichos aditivos se añaden durante el proceso de desalinización y se descargan en las aguas costeras como contaminantes (Lattemann y Höpner 2007).

Las características del agua de rechazo dependen de la calidad del agua de alimentación de la planta desalinizadora, la calidad del agua producida, el método de pretratamiento y los procedimientos de limpieza empleados (Cuadro I). Las concentraciones de sales en las aguas de rechazo suelen ser el doble que en el agua de alimentación, o incluso mayores (Pérez 2014). Lo que favorece la generación de una actividad extra al proceso de desalinización, como la instalación de una granja acuícola en las cercanías de la planta desalinizadora para la producción de camarones en estanques de alto valor comercial que aporten empleo a las comunidades.

La gestión tradicional de los concentrados procedentes de plantas de desalinización está principalmente condicionada por la localización de las mismas. En plantas de desalinización costera, los concentrados son vertidos directamente al mar, 
CUADRO I. CONCENTRACIONES DE AGUA DE RECHAZO SEGÚN PROCESOS DE DESALINIZACIÓN

\begin{tabular}{|c|c|c|c|c|}
\hline \multirow{2}{*}{\multicolumn{2}{|c|}{$\begin{array}{l}\text { Concentración de agua de } \\
\text { alimentación (mg/L de SDT) }\end{array}$}} & \multicolumn{3}{|c|}{$\begin{array}{l}\text { Concentración de salmuera según tecnología empleada } \\
\qquad(\mathrm{mg} / \mathrm{L} \text { de SDT })\end{array}$} \\
\hline & & \multirow{2}{*}{$\frac{\text { BWRO }}{2-2000}$} & \multirow{2}{*}{$\frac{\text { SWRO }}{2-2000}$} & \multirow{2}{*}{$\frac{\text { BWED }}{2-2000}$} \\
\hline Agua de red & $1-1000$ & & & \\
\hline Agua salobre & $1000-10000$ & $2000-20000$ & $2000-20000$ & $2000-20000$ \\
\hline Agua salina & $10000-35000$ & ---- & $20000-60000$ & ---- \\
\hline Agua de mar & $>35000$ & ---- & $65000-80000$ & ---- \\
\hline
\end{tabular}

Nomenclatura: BWRO = ósmosis inversa para agua salobre (brackish water reverse osmosis), SWRO = ósmosis inversa para agua de mar (sea water reverse osmosis), BWED = electrodiálisis para agua salobre (brackish water electrodialysis), $\mathrm{SDT}=$ sólidos disueltos totales

Fuente: Medina (2000), Dévora (2006)

mientras que en plantas de desalinización situadas tierra adentro, las opciones de gestión tradicionales consisten en reducir el volumen del concentrado como etapa previa al vertido (Pérez 2014).

A nivel mundial no existe normatividad vigente referente al proceso de desalinización de aguas marinas y salobres, que regule o controle los impactos ambientales al medio marino que pudiesen generar sus productos y desechos (González y Dévora 2009). Sin embargo, en México existe un anteproyecto de Norma Oficial Mexicana, propuesto y elaborado por el Instituto Tecnológico de Sonora. En él se establece un control eficaz de los vertidos de salmueras y otros contaminantes residuales provenientes de plantas desalinizadoras para prevenir y/o mitigar impactos en los ecosistemas acuáticos receptores.

Referente a los técnicas de disposición, se ha encontrado en la literatura que existen distintos métodos convencionales de disposición de salmueras producidas por plantas desalinizadoras. En este artículo se presenta una breve descripción de los métodos más utilizados y posteriormente se presenta en el cuadro II las ventajas y desventajas de los mismos.

Eliminación en aguas superficiales. Este método de tratamiento consiste en la descarga del concentrado en una masa de agua superficial, es decir al océano cercano o al mar, ríos, estuarios, bahías o lagos. Es la práctica más común, ya que la mayoría de las plantas están situadas junto a las aguas superficiales (WHO 2007, Bleninger et al. 2010).

Descargas al alcantarillado. Este método sólo es adecuado para la eliminación de concentrado desde plantas de aguas salobres muy pequeñas. La descarga de concentrado a la alcantarilla sanitaria está regulada por las disposiciones aplicables a las descargas industriales y los reglamentos aplicables de descarga que se encargan de la gestión del sistema de alcantarillado sanitario (López 2014).
Pozo de inyección profunda. Consiste en la inyección de la salmuera en un acuífero profundo confinado, por debajo de un acuífero de agua dulce utilizando un sistema de pozos de eliminación. Este conjunto de pozos también sirven para confirmar que el concentrado no se propague a los acuíferos adyacentes, por lo que se requiere de condiciones geológicas adecuadas (WHO 2007).

Estanques de evaporación. Se basa en la evaporación solar natural de la salmuera en estanques de tierra alineados u otras cuencas (López 2014).

Aplicación en suelo. Permite la reutilización de efluentes de desalinización para el riego de cultivos tolerantes a la salinidad. Depende de la tolerancia de las plantas a la salinidad y del cumplimiento con las normas de calidad para agua de riego (Bleninger et al. 2010).

Cero descarga de líquidos. Estas tecnologías son aquellas capaces de separar el concentrado en agua altamente purificada y el agua de rechazo en un producto sólido seco adecuado para la eliminación en vertederos o la recuperación de sales útiles, como concentradores, cristalizadores y secadores de salmuera (Bleninger et al. 2010).

Granja acuícola. Conjunto de instalaciones dedicadas al cultivo y producción de recursos acuícolas, que permite la reutilización de salmueras obtenidas en el proceso de desalinización (18 000 a $22000 \mathrm{mg} / \mathrm{L}$ ), siempre y cuando la alimentación sea proveniente de agua salobre preferentemente en concentraciones de 9000 a $11000 \mathrm{mg} / \mathrm{L}$ (Ceballos 2012).

El objetivo de este estudio es realizar una comparación de técnicas de descarga para vertidos de salmueras producidas por una planta desalinizadora. Lo anterior a través de la investigación de métodos de disposición adecuados como descargas en aguas superficiales, descargas al alcantarillado, inyección en pozos profundos, estanques de evaporación, 
CUADRO II. MÉTODOS DE DISPOSICIÓN DE SALMUERAS PARA PLANTAS DESALINIZADORAS

\begin{tabular}{lll}
\hline Métodos de disposición & Ventajas & Desventajas \\
\hline $\begin{array}{lll}\text { Descargas en aguas } \\
\text { superficiales }\end{array}$ & - Puede manejar grandes volúmenes. & - Si se excede el límite de capacidad de asimilación \\
& - Los procesos naturales promueven la & $\begin{array}{l}\text { natural, puede causar impactos adversos sobre el medio } \\
\text { ambiente acuático. }\end{array}$ \\
& degradación. & $\begin{array}{l}\text { - La dilución depende de las condiciones hidrodinámi- } \\
\text { cas locales. }\end{array}$ \\
& - Es a menudo la opción menos costosa. & - Se requiere de buen conocimiento y seguimiento de las \\
& aguas receptoras.
\end{tabular}

Descargas al alcantarillado
- Dilución a través de la corriente de residuos.

- Utiliza la infraestructura existente.

- Posible tratamiento beneficioso.

- Capacidad restringida en función de la planta de aguas residuales.

- Debe cumplir con los estándares de calidad de descargas al alcantarillado.

- Su disposición final generalmente es en aguas superficiales.

\begin{tabular}{|c|c|c|}
\hline $\begin{array}{l}\text { Inyección en pozos } \\
\text { profundos }\end{array}$ & $\begin{array}{l}\text { - No ocasiona impactos marinos. } \\
\text { - Es una buena opción para las plantas alejadas } \\
\text { de la costa. }\end{array}$ & $\begin{array}{l}\text { - Viable para plantas de grandes volúmenes. } \\
\text { - Difícil de evaluar su capacidad máxima. } \\
\text { - Requiere adecuada estructura del acuífero. } \\
\text { - Peligro de contaminación del agua subterránea. }\end{array}$ \\
\hline $\begin{array}{l}\text { Estanques } \\
\text { de evaporación }\end{array}$ & $\begin{array}{l}\text { - No ocasiona impactos marinos. } \\
\text { - Posible explotación de sal comercial. } \\
\text { - Requiere de menor gestión y esfuerzos } \\
\text { tecnológicos. }\end{array}$ & $\begin{array}{l}\text { - Capacidad altamente restringida. } \\
\text { - Se requieren grandes extensiones de tierra. } \\
\text { - Sólo puede establecerse en lugares con clima seco y } \\
\text { alta evaporación. } \\
\text { - Riesgo de contaminación del suelo y aguas subterrá- } \\
\text { neas. } \\
\text { - Es necesaria la eliminación de sales inutilizables }\end{array}$ \\
\hline Aplicación en suelo & $\begin{array}{l}\text { - No ocasiona impactos a flora y fauna marina. } \\
\text { - Fuente de agua alternativa para riego de } \\
\text { especies tolerantes a la salinidad (Salicornia, } \\
\text { Remolacha, Cebada, etc.). }\end{array}$ & $\begin{array}{l}\text { - Sólo para flujos de descargas pequeñas. } \\
\text { - Impacto adverso de los productos químicos y contami- } \\
\text { nantes en flora y fauna terrestre. } \\
\text { - Riesgo de contaminación del suelo y aguas subterrá- } \\
\text { neas. } \\
\text { - Sistema de almacenamiento y distribución necesario. }\end{array}$ \\
\hline $\begin{array}{l}\text { Cero descarga de } \\
\text { líquidos }\end{array}$ & $\begin{array}{l}\text { - No hay desechos líquidos. } \\
\text { - Recuperación de sal y minerales. }\end{array}$ & $\begin{array}{l}\text { - Aún no es viable a escala industrial. } \\
\text { - Genera residuos sólidos. } \\
\text { - Requiere de alta energía y costo elevado. }\end{array}$ \\
\hline Granja acuícola & $\begin{array}{l}\text {-Alternativa viable para el uso de aguas no } \\
\text { aptas para la agricultura. } \\
\text {-Abastecimiento para consumo local. } \\
\text {-No se vierte en cuerpos de agua. } \\
\text {-Beneficios económicos. } \\
\text {-Limita enfermedades (Mancha Blanca, } \\
\text { Mancha Amarilla) presentes en el agua de mar. }\end{array}$ & $\begin{array}{l}\text {-Requiere de instalación rigurosa para el cuidado de } \\
\text { infiltraciones al acuífero, además de monitoreo en el } \\
\text { recambio de agua. } \\
\text {-Fugas en la instalación puede causar efectos adversos } \\
\text { sobre la ecología y agricultura cercanas a la misma. } \\
\text {-El costo de instalación es elevado. }\end{array}$ \\
\hline
\end{tabular}

Fuente: Alameddine y ElFadel (2007) y Ceballos J. et al. (2012)

aplicación en suelo, cero descargas de líquidos y granja acuícola, para proponer la técnica más apropiada que disminuya el impacto ambiental producido en la zona de estudio.

\section{MATERIALES Y MÉTODOS}

\section{Localización de la zona de estudio}

El estudio se desarrolló en el campo experimental
1814, de la zona agrícola del Valle del Yaqui, en Ciudad Obregón, Sonora, México (10952'16.6”W; $27^{\circ} 11$ '21.1’N; Fig. 1).

\section{Operación de planta desalinizadora $100 \mathrm{~m}^{3} / \mathrm{d}$}

Se operó una planta desalinizadora por OI con capacidad nominal de $100 \mathrm{~m}^{3} / \mathrm{d}(85 \%$ producto y $15 \%$ rechazo), compuesta por ocho módulos de membranas comerciales de la empresa Nitto Denko de Hydranautics, modelo SWC4 de 8"× 40". Esta agua 




Fig. 1. Localización geográfica de la zona de estudio

fue utilizada en el riego del cultivo de sorgo (Sorghum spp.) en el periodo de junio-octubre 2013 y en el cultivo de tomatillo (Physalis ixocarpa brot.) variedad Gran Esmeralda, en el periodo de diciembre-marzo 2015, etapas donde se obtuvieron resultados históricos de la operación de la planta desalinizadora que sirven como marco de referencia. El pretratamiento físico cuenta con un filtro multimedia compuesto de arena, antracita y grava para partículas suspendidas de $50 \mu \mathrm{m}$. Posteriormente, el agua de alimentación del pozo pasa por cinco filtros de cartucho de $5 \mu \mathrm{m}$. En el pretratamiento químico se utilizó una bomba de pulsaciones para dosificación de antiescalante de 0.1 caballos de fuerza, (HP, por sus siglas en inglés). Se utilizaron $3 \mathrm{~L}$ de antiescalante y que fueron aforados hasta 30 L (concentración del $10 \%$ ). El caudal se alimentó a una razón de $2.97 \mathrm{~mL} / \mathrm{min}$, con $30 \%$ de pulsaciones y $30 \%$ de frecuencia. El antiescalante fue Flocon Plus N, de BWA Water Additives. El costo de dosificación fue de $0.06 \mathrm{MX} \$ / \mathrm{m}^{3}$.

Se realizó toma de muestras de agua de alimentación proveniente de un pozo de agua salobre y de agua de rechazo producida por la planta desalinizadora durante los periodos de tiempo señalados, con la finalidad de caracterizar las aguas y comparar la diferencia de concentraciones entre muestras.

\section{Medición de parámetros de calidad del agua}

Como parámetros de campo se midieron, tanto en muestras del pozo de alimentación al proceso como en el rechazo de la planta desalinizadora, las siguientes variables: conductividad eléctrica $(\mathrm{mS} / \mathrm{cm})$, SDT (mg/L), oxígeno disuelto (OD en \%), pH y temperatura $\left({ }^{\circ} \mathrm{C}\right)$. Para ello se utilizó un equipo de medición multiparamétrico modelo YSI 556 MPS (Sistema Multi-Sonda), resistente al agua (satisface las especificaciones IP67). Cuenta con modulo de electrodos de campo sustituibles y sensores de OD, $\mathrm{pH}$ o de $\mathrm{pH} /$ potencial óxido-reducción (ORP, por sus siglas en inglés).

Posteriormente, se analizaron las concentraciones de los parámetros físicos y químicos en $\mathrm{mg} / \mathrm{L}$ de iones calcio $\left(\mathrm{Ca}^{++}\right)$, magnesio $\left(\mathrm{Mg}^{++}\right)$, sodio $\left(\mathrm{Na}^{+}\right)$, potasio $\left(\mathrm{K}^{+}\right)$, bicarbonato $\left(\mathrm{HCO}_{3}{ }^{-}\right)$, sulfato $\left(\mathrm{SO}_{4}{ }^{-}\right)$y cloruro $\left(\mathrm{Cl}^{-}\right)$en el agua de alimentación y en el agua de rechazo.

\section{Límites máximos permisibles en descargas de salmueras}

Se compararon los resultados obtenidos con el Anteproyecto de Norma Oficial Mexicana (PROYNOM-013-CONAGUA/SEMARNAT-2015), que establece las especificaciones y requisitos para las obras de toma y descarga que se deben cumplir en las plantas desalinizadoras o procesos que generen aguas de rechazo salobres o salinas, para determinar si los parámetros medidos en agua de rechazo cumplen con los límites máximos permisibles según el cuerpo receptor de su descarga actual.

\section{Planes de manejo de vertidos de salmuera}

Se consultaron métodos de disposición de vertidos de salmuera (Lattemann y Höpner 2007) a nivel internacional para determinar los más convenientes. Lo anterior, dependiendo de la tecnología utilizada en el proceso de desalinización, de las características de las plantas desalinizadoras y de sus vertidos, así como de la naturaleza física y de las condiciones 
CUADRO III. RESULTADOS DE ANÁLISIS EN VARIABLES DE CAMPO EN MUESTRAS DE AGUA

\begin{tabular}{|c|c|c|c|c|c|}
\hline & $\mathrm{pH}$ & $\begin{array}{c}\text { Temperatura } \\
\left({ }^{\circ} \mathrm{C}\right)\end{array}$ & $\begin{array}{c}\text { Conductividad } \\
\text { eléctrica }(\mathrm{mS} / \mathrm{cm})\end{array}$ & $\begin{array}{l}\text { Oxígeno } \\
\text { disuelto }\end{array}$ & SDT (mg/L) \\
\hline \multicolumn{6}{|c|}{ Periodo junio - octubre 2013} \\
\hline Alimentación & $7.42 \pm 0.21$ & $25.50 \pm 0.61$ & $10.43 \pm 0.15$ & --- & $6610.20 \pm 97.16$ \\
\hline Rechazo & $7.69 \pm 0.17$ & $29.57 \pm 3.26$ & $35.34 \pm 3.25$ & --- & $21300.00 \pm 2728.25$ \\
\hline \multicolumn{6}{|c|}{ Periodo diciembre 2014 - marzo 2015} \\
\hline Alimentación & $7.39 \pm 0.05$ & $25.90 \pm 0.38$ & $4.58 \pm 0.11$ & $2.94 \pm 2.29$ & $2931.21 \pm 76.45$ \\
\hline Rechazo & $7.68 \pm 0.04$ & $26.63 \pm 1.11$ & $10.55 \pm 0.30$ & $2.46 \pm 1.79$ & $6888.80 \pm 196.92$ \\
\hline
\end{tabular}

SDT $=$ sólidos disueltos totales, \pm error estándar

biológicas del ambiente receptor (Fernández 2012). Una vez analizado el comparativo entre ventajas y desventajas, se propone un método alternativo para control y uso de los vertidos de salmueras según las condiciones locales del proyecto.

\section{RESULTADOS}

\section{Calidad de agua en planta desalinizadora de ós- mosis inversa}

En el cuadro III, se muestra el promedio y desviación estándar de los parámetros de calidad del agua. En el agua de alimentación proveniente del pozo se encontraron valores de conductividad eléctrica de $10.43 \pm 0.15$ y $4.58 \pm 0.11$, mientras que para el agua de rechazo, los valores fueron de 35.34 \pm 3.25 y $10.55 \pm 0.30$, para los periodos de junio octubre 2013 y de diciembre 2014 - marzo 2015, respectivamente.

La figura 2 muestra el comportamiento de SDT y la figura 3 el comportamiento de $\mathrm{pH}$, ambas figuras en parámetros medidos en agua de alimentación y en agua de rechazo en los dos periodos de habilitación de la planta desalinizadora. Se observa que en el agua de alimentación proveniente del pozo en el periodo de diciembre 2014 a marzo 2015, se presentó una disminución del $44.34 \%$ de SDT (de 6610.20 a $2931.21 \mathrm{mg} / \mathrm{L}$ ), respecto al periodo de junio a octubre 2013.

Por otra parte, en los diagramas de cajas no se observan diferencias significativas en el $\mathrm{pH}$ de los dos periodos de trabajo. El proceso de desalinización no afecta al $\mathrm{pH}$ en las corrientes de agua de alimentación y de rechazo, sólo afecta al agua producto, por lo que se debe hacer una remineralización.

\section{Comparación de vertidos de salmuera respecto al anteproyecto de NOM}

Para la descarga de aguas, es necesario cumplir con los límites máximos permisibles establecidos, dependiendo del cuerpo receptor. El cuadro IV muestra la comparación de resultados de agua de rechazo de la planta desalinizadora de $100 \mathrm{~m}^{3} / \mathrm{d}$
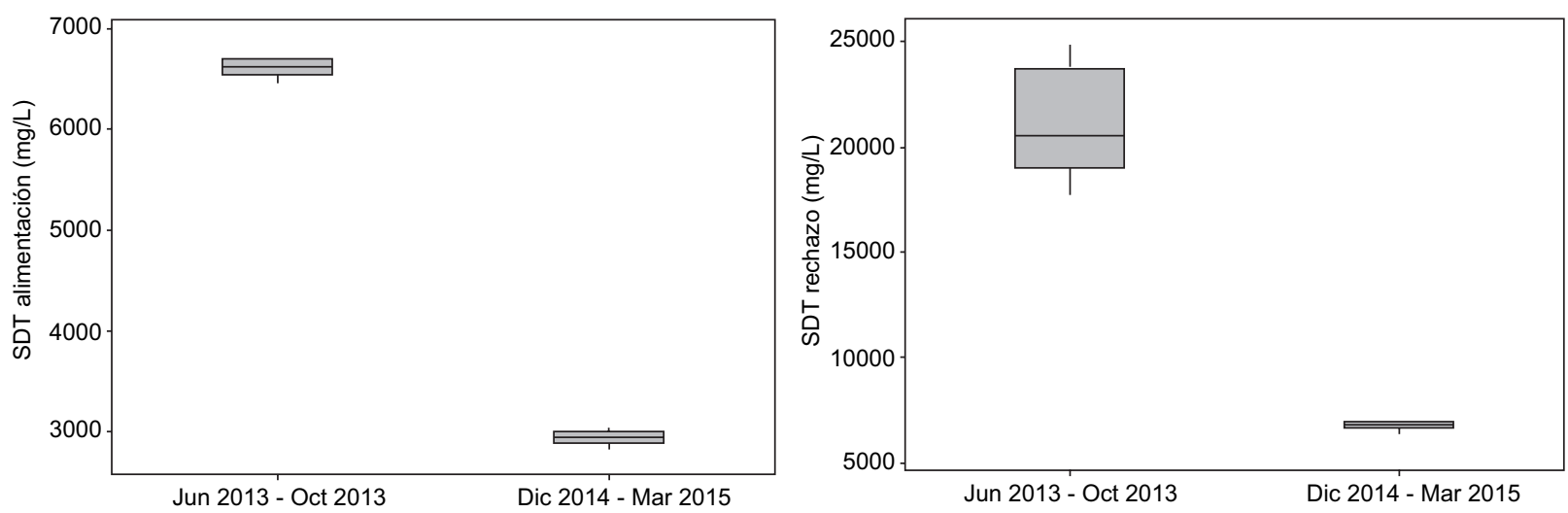

Fig. 2. Comparación de sólidos disueltos totales (SDT) en agua de alimentación y en agua de rechazo en los dos periodos evaluados. Las barras indican la desviación estándar presente en las muestras 



Fig. 3. Comparación de $\mathrm{pH}$ en agua de alimentación y en agua de rechazo en los dos periodos evaluados. Las barras indican la desviación estándar presente en las muestras

CUADRO IV. COMPARACIÓN DEL VERTIDO DE SALMUERA CONTRA ANTEPROYECTO DE LA NORMA OFICIAL MEXICANA (NOM)

\begin{tabular}{lrrrrrrrr}
\hline \multirow{2}{*}{ Muestra } & \multicolumn{8}{c}{$(\mathrm{mg} / \mathrm{L})$} \\
\cline { 2 - 9 } & \multicolumn{1}{c}{$\mathrm{Ca}^{++}$} & \multicolumn{1}{c}{$\mathrm{Mg}^{++}$} & $\mathrm{Na}^{+}$ & \multicolumn{1}{c}{$\mathrm{K}^{+}$} & $\mathrm{HCO}_{3}{ }^{-}$ & \multicolumn{1}{c}{$\mathrm{SO}_{4}{ }^{-}$} & $\mathrm{Cl}^{-}$ & SDT \\
\hline Propuesta NOM & 20.00 & 80.00 & 650.00 & 20.00 & 10.00 & 400.00 & 1000.00 & 2000.00 \\
Rechazo & 601.00 & 360.00 & 495.00 & 7.60 & 2196.00 & 2192.20 & 1037.00 & 6888.80 \\
\hline
\end{tabular}

SDT $=$ sólidos disueltos totales

contra los límites establecidos cuando esta agua se vierte en cuerpos receptores (como alcantarillado público en zona agrícola), según el anteproyecto de Norma (PROY-NOM-013-CONAGUA/SEMARNAT-2015).
Propuesta para control y uso de vertidos de salmuera

Para este proyecto en particular, después del análisis de ventajas y desventajas, se encontró que una granja acuícola para el cultivo de la especie de

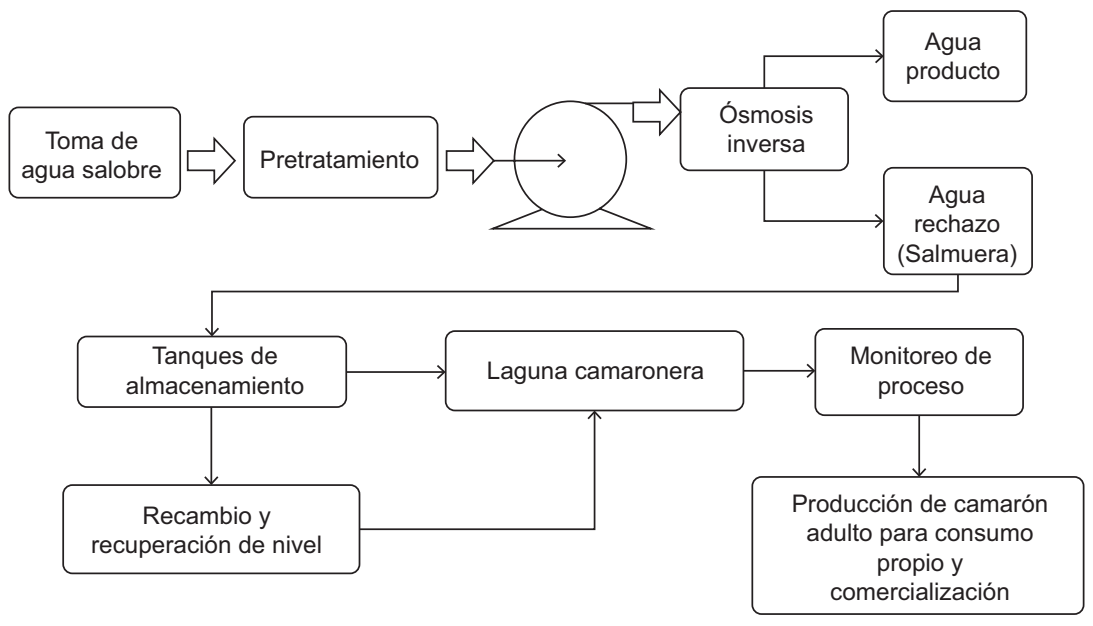

Fig. 4. Diagrama del proceso de operación de una granja acuícola 
camarón blanco (Litopenaeus vannamei) es una buena opción, dado que se adapta a las condiciones del sitio de estudio (Fig. 4).

\section{DISCUSIÓN}

Calidad de agua en planta desalinizadora de ósmosis inversa Agua de alimentación

Los valores de agua de alimentación de este artículo en el periodo de diciembre 2014 - marzo 2015 $(2931.21 \pm 76.45 \mathrm{mg} / \mathrm{L})$, coinciden con otros proyectos a nivel mundial en procesos de desalinización de agua salobre por OI en Wadi Ma'in, Jordania, $(1200-2000 \mathrm{mg} / \mathrm{L})$. Asimismo, concuerdan con estudios de una planta desalinizadora en El Paso Texas, EUA, cuyos valores fueron de $2550 \mathrm{mg} / \mathrm{L}$ (IDA 2007).

\section{Agua de rechazo}

Los parámetros medidos de calcio $\left(\mathrm{Ca}^{++}\right)$, magnesio $\left(\mathrm{Mg}^{++}\right)$, bicarbonatos $\left(\mathrm{HCO}_{3}^{-}\right)$, sulfatos $\left(\mathrm{SO}_{4}{ }^{-}\right)$, cloruros $\left(\mathrm{Cl}^{-}\right)$y SDT muestran en su mayoría, valores más elevados en agua de rechazo respecto al agua de alimentación. Para este proyecto, las concentraciones de los parámetros obtenidos en agua rechazo se encuentran fuera del rango permisible en el anteproyecto de NOM (Cuadro IV), por lo que se debe considerar un método de disposición adecuado para disminuir el impacto ambiental que estas aguas ocasionan (Dévora 2013).

\section{Comportamiento de concentración en agua de alimentación y de rechazo}

El parámetro de SDT medido en el periodo de junio - octubre 2013 y diciembre de 2014 - marzo 2015 , muestra diferencias relevantes en cuanto a una disminución de concentración. Esto puede deberse a un incremento del flujo de agua dulce en el acuífero producido por las lluvias durante el último periodo en la región, siendo mayor el volumen de recarga que el de extracción de agua del pozo de estudio (Morell y Hernández 2000). Sin embargo, se debe prestar atención especial al monitoreo del acuífero, debido a que en periodos secos, las extracciones de agua subterránea podrían exceder el volumen de recarga, disminuyendo el flujo de agua dulce e incrementando el nivel de intrusión salina. Lo anterior aumenta a su vez la cantidad de SDT en el agua de alimentación y en el agua de rechazo producida por la planta desalinizadora. Esto puede convertirse en un riesgo ambiental ya que, según Bleninger et al. (2010), el aumento de las concentraciones de sal pueden ser perjudiciales e incluso letales para la vida acuática. En esta fase los métodos de disposición de salmueras toman importancia, ya que el origen y caracterización química de los concentrados es un factor clave para la selección del método más adecuado.

\section{Sistemas de disposición de salmueras}

El conocer la manera de disposición de salmueras es una buena alternativa para el uso de los subproductos del proceso de desalación, disminuyendo el impacto ambiental que éstos ocasionan. Sin embargo, de los métodos propuestos, no se puede especificar uno de ellos que aplique para todos los escenarios, ya que la disposición de las aguas de rechazo depende del proceso utilizado (sistemas de membranas o sistemas térmicos) y del tamaño de la planta desalinizadora. Por lo tanto, es necesario considerar la gestión de esta agua de rechazo como una parte integral en el diseño de cualquier instalación (MSPS 2009). Bleninger et al. (2010) mencionan que desafortunadamente los sistemas de descargas de salmuera a menudo no se sitúan correctamente y no se encuentran del todo optimizados respecto a las condiciones de mezcla y la distribución de sustancias (Dévora 2013). Lo que conduce a impactos ambientales innecesarios o incluso problemas de funcionamiento de la planta desalinizadora (Lattemann 2010).

\section{Control y uso de vertidos de salmuera}

En la figura 4, se muestra el diagrama del proceso de operación de la granja acuícola, tomando en cuenta los aspectos técnicos necesarios para su construcción desde el inicio del proyecto. En investigaciones previas, autores reportan que para el cultivo de camarón Litopenaeus vannamei en agua de mar, se toma como referencia la salinidad entre $32000 \mathrm{y}$ $35000 \mathrm{mg} / \mathrm{L}$ (Dévora 2009). En cuanto al agua de rechazo de este estudio, es importante resaltar que su salinidad es entre 6500 y $7000 \mathrm{mg} / \mathrm{L}$. Sin embargo, se conoce que el camarón es una especie eurihalina (resistente a grandes cambios de salinidad, que van desde aguas salobres en esteros hasta ambientes marinos). Bajo este concepto, la implementación de esta propuesta generará una ganancia externa con el producto obtenido en la granja acuícola y a su vez minimizará el impacto ambiental ocasionado por los vertidos de salmuera.

Es importante que las granjas acuícolas cuenten con mantenimiento adecuado de sus instalaciones para evitar fugas que provoquen infiltraciones al acuífero. Cuando se recambia el agua y se retira la sal del proceso, se capturarán las aguas de desecho 
en recipientes confinados para su disposición final, las cuales se trasladarán a una salinera de la región. Con esta acción se evitarían las descargas directas al ambiente.

\section{CONCLUSIÓN}

El objetivo de este trabajo se cumplió cabalmente al realizarse una comparación entre técnicas de descarga para vertidos de salmuera y establecerse una propuesta aplicada al agua de rechazo del proceso de desalinización. Esta propuesta consistió en la reutilización de los vertidos de salmuera para un proceso de cultivo de camarón en estanques en ambiente controlado, derivado de las condiciones naturales de la zona de estudio.

Por otra parte, los métodos de disposición de salmueras encontrados en literatura señalan que los más importantes son: descargas en aguas superficiales, descargas al alcantarillado, inyección en pozos profundos, estanques de evaporación, aplicación en suelo, cero descargas de líquidos y granja acuícola.

Bajo este contexto, es importante regular o disponer el vertido de salmuera para evitar impactos negativos al ecosistema receptor por su alta concentración. Es importante señalar que la magnitud de los efectos producidos por los vertidos de salmuera serán distintos según el tamaño y tipo de planta desalinizadora. Por lo anterior, la información generada en este estudio es útil, para que futuros proyectos de desalinización se sometan a estudios rigurosos de impacto ambiental que favorezcan la minimización de los efectos negativos del proceso y opten por una de las propuestas aquí presentadas.

\section{AGRADECIMIENTOS}

Los autores agradecen el financiamiento otorgado por la Fundación PRODUCE Sonora, A. C., para llevar a cabo este proyecto de investigación. Asimismo se agradece al Ing. Fructuoso Alejandro Méndez, quien facilitó el terreno agrícola y el uso de pozo salobre para operar la planta desalinizadora.

\section{REFERENCIAS}

Alameddine I. y El-Fadel M. (2007). Brine discharge from desalination plants: a modeling approach to an optimized outfall design. Desalination 214 (1-3), 241260. DOI: 10.1016/j.desal.2006.02.103
Bleninger T. y Jirka G. (2010). Environmental planning, prediction and management of brine discharges from desalination plants [en línea]. http://www.ifh.uni-karlsruhe.de/science/envflu/research/brinedis/ 15/05/2015.

Ceballos J., Cabrera J. y Vega F. (2012). Cultivo tierra adentro de camarón marino Litopenaeus vannamei: evaluación del agua de dos granjas acuícolas en Cuba. Revista Electrónica de Veterinaria 13, 1-17.

Dévora G. E. (2006). Diseño de procesos de desalinización de aguas subterráneas salobres mediante simulación química de electrodiálisis reversible, con propósito de consumo humano. Revista Latinoamericana de Recursos Naturales 5 (1), 31-41.

Dévora G. E. (2009). Toxicological bioassays in white shrimp (Litopenaeus Vannamei) by desalination plant brine. IDA World Congress. Memoria. Dubai, Emiratos Árabes Unidos. 7 al 12 de noviembre, pp. 76-86.

Dévora G. E., González R., Meza E. y Saldivar J. (2012). Evaluación de una fuente de agua de mar para la implementación de una desaladora. Ide@s CONCYTEG 7 (85), 923-944.

Dévora G., González R. y Milian J. (2013). Localización de obra de toma de agua de mar, para instalación de una desaladora en Guaymas, Sonora. En: Desalación (G. E. Dévora, R. González y J. Saldivar, Eds.). Instituto Tecnológico de Sonora, Sonora, México, pp. 60-85.

Dévora G. E., González R. y Ruiz S. (2013). Evaluación de procesos de desalinización y su desarrollo en México. Tecnología y Ciencias del Agua 4 (3), 27-46.

Fernández Y. (2012). Efectos de las variaciones de salinidad sobre angiospermas marinas y su aplicación a los vertidos de plantas desalinizadoras. Tesis de Doctorado. Universidad de Alicante, España, 252 pp.

Gacía E. y Ballesteros E. (2001). El impacto de las plantas desalinizadoras sobre el medio marino: la salmuera en las comunidades bentónicas mediterráneas [en línea]. http://www3.uah.es/tiscar/Complem_EIA/impactodesaladoras.pdf 13/05/2015.

González E.R. y Dévora G.E. (2009). Funcionamiento y contaminación generada por plantas desalinizadoras ubicadas en las zonas del mar de Cortés y mar Caribe: un estudio para el desarrollo de normatividad ambiental acuática. Revista Latinoamericana de Recursos Naturales 5 (2), 186-197.

IDA (2007). Desalination yearbook. 1a ed. International Desalination Asociation. Oxford, Inglaterra, 208 pp.

Lattemann S. y Höpner T. (2007). Environmental impact and impact assessment of seawater. Desalination 220 (1-3), 1-15. DOI: 10.1016/j.desal.2007.03.009

Lattemann S., Kennedy M. D., Schippers J. C. y Amy G. (2010). Chapter 2 Global desalination situation. Sustainability Science and Engineering 2, 7-39.

DOI: $10.1016 / \mathrm{S} 1871-2711(09) 00202-5$ 
López M. E. (2013). Evaluación de una planta desalinizadora de ósmosis inversa para su empleo en agricultura. Tesis de Licenciatura. Instituto Tecnológico de Sonora. Ciudad Obregón, Sonora, México, 50 pp.

López M. G. (2014). Evaluación de un destilador solar tipo invernadero asistido con telas de evapotranspiración para la obtención de agua potable a partir de agua de mar. Tesis de Maestría. Universidad Nacional Autónoma de México. Ciudad de México, México, 98 pp.

Medina J. (2000). Desalinización de aguas salobres y de mar en ósmosis inversa. Mundi Prensa, Madrid, España, 396 pp.

MSPS (2009). Guía de desalación: aspectos técnicos y sanitarios en la producción de agua de consumo humano. Ministerio de Sanidad y Política Social. Madrid, España, 206 pp.
Morell I. y Hernández F. (2000). El agua en Castellón: un reto para el siglo XXI. Universitat Jaume I. Castellón de la Plana, España, 538 pp.

Morillo J., Usero J., Rosado D., El Bakouri H., Riaza A. y Bernaola F. J. (2014). Comparative study of brine management technologies for desalination plants. Desalination 336, 32-49. DOI: 10.1016/j.desal.2013.12.038

Pérez A. (2014). Tratamiento integrado y valorización de concentrados salobres de ósmosis inversa. Tesis de Doctorado, Universidad de Cantabria. Santander, España, 186 pp.

WHO (2007). Desalination for safe water supply, guidance for the health and environmental aspects applicable to desalination. World Health Organization, Ginebra, Suiza, 173 pp. 